July 1997

rev. Oct. 1997

IFP-742-UNC

VPI-IPPAP-97-5

\title{
On Radiative CP Violation in (Supersymmetric) Two Higgs Doublet Models
}

\author{
Otto C. W. Kong ${ }^{a *}$ and Feng-Li Lin $^{b \dagger}$ \\ ${ }^{a}$ Institute of Field Physics, Department of Physics and Astronomy, \\ University of North Carolina, Chapel Hill, NC 27599-3255. \\ ${ }^{b}$ Department of Physics, and Institute for Particle Physics and Astrophysics, \\ Virginia Polytechnic Institute and State University, Blacksburg, VA 24061-0435.
}

\begin{abstract}
We discuss the feasibility of spontaneous CP violation being induced by radiative corrections in $2 \mathrm{HDM}$ 's, emphasizing on a consistent treatment at 1-loop level. Specifically, we analyze the cases of gaugino/higgsino effect on MSSM, and a new model proposed here with an extra, exotic, quark doublet. The latter model is phenomenologically interesting. One conclusion is that some fine tuning is in general needed for the scenario to work. The case for the new model requires a tree level mass mixing between the two Higgses, which fits in the standard SUSY picture. The fine tuning requires is then very moderate in magnitude and in a way natural.
\end{abstract}

Typeset using REVTEX

\footnotetext{
*Present address: Department of Physics and Astronomy, University of Rochester, Rochester NY 14627-0171. E-mail: kong@pas.rochester.edu

${ }^{\dagger}$ E-mail: linfl@vt.edu
} 
Introduction. The source of $\mathrm{CP}$ violation is one of the most important unsolved puzzles in particle physics. The possibility of $\mathrm{CP}$ being a spontaneously broken symmetry keeps generating new interest. The most simple setting for achieving the scenario is in a two-Higgsdoublet model (2HDM), originally analyzed by Lee [1]. However, in order to avoid flavorchanging-neutral-currents that could result, extra structure like natural flavor conservation (NFC) [2] has to be imposed on a 2HDM, which then forbids spontaneous CP violation (SCPV) [3]. A supersymmetric version of the standard model (SM) is naturally a $2 \mathrm{HDM}$ with NFC being imposed automatically by the holomorphy of the superpotential. Hence, the bulk of study on the minimal supersymmetric standard model (MSSM) concentrates on the CP conserving vacuum. Recently, Maekawa [4] illustrated that SCPV could actually arise in the model in some region of the parameter space through radiative correction. In a subsequent paper, Pomarol [5] argued that mass for the "psuedoscalar", $m_{A}$, is roughly proportional to $\left(\lambda_{5}\right)^{1 / 2}$ and is at least more than a factor of three too small to be phenomenologically acceptable. Both analyses, however, are in a way oversimplified. For instance, the $m_{A}$ mass estimate is, strictly speaking, obtained from a Higgs mass matrix of negative determinant, hence indicating the whole picture is liable to be totally inconsistent. In this letter, we look into the situation more carefully and try to address the various issues involved in a radiative SCPV model of the kind, with or without supersymmetry (SUSY). The basic idea here is that in order to have a consistent approximation to the radiative corrections in whatever interesting region of the parameter space, loop contributions to all parameters in the scalar potential at the same order have to be considered. Our result illustrates that, the radiative $\mathrm{CP}$ violation scenario does not get around the Georgi-Pais theorem [6] in the way claimed in the literature. Specifically, fine tuning is in general needed for it to work.

Despite the Georgi-Pais theorem and the $m_{A}$ mass limit constraint the radiative $\mathrm{CP}$ violation scenario still generate interest $\mathrm{f}$. In our opinion, a realistic model implementing

*See Refs. [7,8], for example. 
the mechanism, hence realizing $\mathrm{CP}$ violation as a pure quantum mechanical effect, is of general interest. It provides a natural way of explaining the smallness of CP violation and a potentially different phenomenology of the latter. Within the domain of a supersymmetric 2HDM specifically, it also provides the only possible scenario of SCPV. Such a model is constructed and presented here. Our new model, has an extra, exotic, quark doublets. It could be phenomenologically-viable, in the sense that $\lambda_{5}$ resulted could easily be more than an order of magnitude larger, hence circumventing the likely small $m_{A}$ obstacle, though the full phenomenological features of the model still have to be worked out. Unlike a recent model using right-handed neutrinos [7], our model achieves a large $\lambda_{5}$ without a fine tuning in the fermion (quark) masses, and is fully compatible with SUSY. However, the same type of fine tuning, though quite moderate numerically, as in the MSSM case is needed to really have radiative $\mathrm{CP}$ violation. We expect this feature to be generic.

The scalar potential and $S C P V$. The most general two Higgs doublet potential is given by

$$
\begin{aligned}
V\left(\phi_{1}, \phi_{2}\right)= & m_{1}^{2} \phi_{1}^{\dagger} \phi_{1}+m_{2}^{2} \phi_{2}^{\dagger} \phi_{2}-\left(m_{3}^{2} \phi_{1}^{\dagger} \phi_{2}+\text { h.c. }\right) \\
& +\lambda_{1}\left(\phi_{1}^{\dagger} \phi_{1}\right)^{2}+\lambda_{2}\left(\phi_{2}^{\dagger} \phi_{2}\right)^{2}+\lambda_{3}\left(\phi_{1}^{\dagger} \phi_{1}\right)\left(\phi_{2}^{\dagger} \phi_{2}\right) \\
& +\lambda_{4}\left(\phi_{1}^{\dagger} \phi_{2}\right)\left(\phi_{2}^{\dagger} \phi_{1}\right)+\frac{1}{2}\left[\lambda_{5}\left(\phi_{1}^{\dagger} \phi_{2}\right)^{2}+\text { h.c. }\right] \\
& +\frac{1}{2}\left\{\phi_{1}^{\dagger} \phi_{2}\left[\lambda_{6}\left(\phi_{1}^{\dagger} \phi_{1}\right)+\lambda_{7}\left(\phi_{2}^{\dagger} \phi_{2}\right)\right]+\text { h.c. }\right\} .
\end{aligned}
$$

Assuming all the parameters in $V$ being real, and denoting the vacuum expectation values (VEV's) of the neutral components of the Higgs doublets by

$$
\left\langle\phi_{1}^{0}\right\rangle=v_{1} \quad \text { and } \quad\left\langle\phi_{2}^{0}\right\rangle=v_{2} e^{i \delta}
$$

we have

$$
\begin{aligned}
\langle V\rangle= & m_{1}^{2} v_{1}^{2}+m_{2}^{2} v_{2}^{2}+\lambda_{1} v_{1}^{4}+\lambda_{2} v_{2}^{4}+\left(\lambda_{3}+\lambda_{4}-\lambda_{5}\right) v_{1}^{2} v_{2}^{2} \\
& +2 \lambda_{5} v_{1}^{2} v_{2}^{2} \cos ^{2} \delta-\left(2 m_{3}^{2}-\lambda_{6} v_{1}^{2}-\lambda_{7} v_{2}^{2}\right) v_{1} v_{2} \cos \delta \\
= & M_{1} v_{1}^{2}+M_{2} v_{2}^{2}+\left(p v_{1}^{4}+2 r v_{1}^{2} v_{2}^{2}+q v_{2}^{4}\right)
\end{aligned}
$$




$$
+2 \lambda_{5} v_{1}^{2} v_{2}^{2}(\cos \delta-\Omega)-\frac{m_{3}^{4}}{2 \lambda_{5}}
$$

where

$$
\Omega=\frac{2 m_{3}^{2}-\lambda_{6} v_{1}^{2}-\lambda_{7} v_{2}^{2}}{4 \lambda_{5} v_{1} v_{2}}
$$

and

$$
\begin{aligned}
M_{1} & =m_{1}^{2}+\frac{\lambda_{6} m_{3}^{2}}{2 \lambda_{5}}, \\
M_{2} & =m_{2}^{2}+\frac{\lambda_{7} m_{3}^{2}}{2 \lambda_{5}}, \\
p & =\lambda_{1}-\frac{\lambda_{6}^{2}}{8 \lambda_{5}}, \\
q & =\lambda_{2}-\frac{\lambda_{7}^{2}}{8 \lambda_{5}}, \\
r & =\frac{1}{2}\left(\lambda_{3}+\lambda_{4}-\lambda_{5}-\frac{\lambda_{6} \lambda_{7}}{4 \lambda_{5}}\right) .
\end{aligned}
$$

A nontrivial phase $(\delta)$ then indicates SCPV.

Let us look at the $\delta$-dependence of $\langle V\rangle$. The extremal condition gives

$$
-4 \lambda_{5} v_{1}^{2} v_{2}^{2}(\cos \delta-\Omega) \sin \delta=0
$$

and the stability condition requires

$$
\frac{\partial^{2} V}{\partial \delta^{2}}=4 \lambda_{5} v_{1}^{2} v_{2}^{2}\left[\cos \delta(\Omega-\cos \delta)-\sin ^{2} \delta\right]>0
$$

$\cos \delta=\Omega$ gives a SCPV solution, provided that $\lambda_{5}>0$ and $|\Omega|<1$. Actually, Eq.(2) shows that this is the absolute minimum. Then one can easily obtain the result that

$$
\begin{aligned}
& v_{1}^{2}=\frac{1}{2} \frac{r M_{2}-q M_{1}}{p q-r^{2}} \\
& v_{2}^{2}=\frac{1}{2} \frac{r M_{1}-p M_{2}}{p q-r^{2}}
\end{aligned}
$$

In order for $\mathrm{V}$ to have a lower bound, we have the extra constraints

$$
p>0, \quad q>0
$$


and

$$
p q>r^{2}
$$

which demand that

$$
r M_{2}>q M_{1}, \quad r M_{1}>p M_{2}
$$

Note that if $p>0, q>0$ and $r<0$, then $M_{1} M_{2}<0$ is required for a consistent solution.

On the other hand, for a CP conserving vacuum, $\sin \delta=0$ and the stability condition reads

$$
\pm \lambda_{5}(\Omega \mp 1)>0
$$

with the two signs correspond to the cases $\delta=0$ and $\pi$ respectively. Only one of them would give a minimum in whatever region of the parameter space. For instance, $\delta=\pi$, corresponding to a negative $\tan \beta\left(=v_{2} / v_{1}\right)$, is the only minimum for $\lambda_{5}>0, \Omega<-1$ and $\lambda_{5}<0, \Omega>-1$.

Without SUSY, the natural way to impose NFC is to require that only one of the Higgs, say $\phi_{1}$, transforms nontrivially under an extra symmetry. This means that $m_{3}^{2}, \lambda_{6}$ and $\lambda_{7}$, and may be $\lambda_{5}$ too, all have to vanish. The same result is obtained from the superpotential of the MSSM, except that the soft SUSY breaking $B$-term gives rise to a nonvanishing $m_{3}^{2}$. All these are about the tree level scalar potential. The interesting point of concern here is whether radiative corrections can modify the picture.

Radiative CP violation. For the MSSM case, Maekawa [4] pointed out that there is a positive contributions to $\lambda_{5}$ from a finite 1-loop diagram (Fig. 1a) involving the gauginos and higgsinos, which could lead to SCPV. The small value of $\Delta \lambda_{5}\left(\sim g^{4} / 16 \pi^{2}\right)$ resulting, however, could lead to phenomenological problem [5]. The lesson here may be that extra, probably vector-like, fermions with appropriate mixed couplings to both Higgs doublets could make good candidate models for the radiative $\mathrm{CP}$ violation scenario. The fermions may be gauginos and higgsinos, or right-handed neutrinos [7], or, as illustrated in our new 
model below, quarks. These fermions do lead to flavor changing neutral currents which, however, could be made to be sufficiently suppressed.

Our new model has an extra pair of vectorlike quark doublets, $Q$ and $\bar{Q}$, with the following couplings

$$
\mathcal{L}_{Q}=M_{Q} \bar{Q} Q+\lambda_{Q} \bar{t} \phi_{1}^{\dagger} Q
$$

as an addition to the SM with two Higgs doublets or MSSM. Note that $\phi_{1}^{\dagger}$ is actually $H_{d}$, the Higgs (super)multiplet that gives masses to the down-type quarks; and $\phi_{2}$ is $H_{u}$. So, $T_{3}=-1 / 2$ component of $Q$, denoted by $T$, has the same charge as the top quark and mixes with it after electroweak (EW) symmetry breaking. The other part of the doublet is a quark of electric 5/3. The 1-loop diagram, now with the gaugino/higgsino propagators replaced by that of the quarks, leads to $\Delta \lambda_{5}\left(\sim 3 \lambda_{Q}^{2} \lambda_{t}^{2} / 16 \pi^{2}\right)$ and could be very substantial for large Yukawa couplings. Note that $\phi_{1}$ and $\phi_{2}$ vertices now have $\lambda_{Q}$ and $\lambda_{t}$ couplings respectively. If $M_{Q}$ around the same order as the EW scale, this can easily get around the above mentioned phenomenological objection without fine tuning of the fermion masses, unlike the case with right-handed neutrinos. Modification to top quark phenomenology would then be very interesting. Another interesting point is that the scalar partner of $\bar{Q}$ in the SUSY case has exactly the quantum numbers needed to be a leptoquark that can produce the high- $Q^{2}$ anomaly at HERA [9]. $Q-\bar{Q}$ could naturally arise, for example, as the only extra quarks from some interesting models with a SM-like chiral fermion spectra embedding the three SM families in a intriguing way [10].

A consistent treatment. Nevertheless, a $\Delta \lambda_{5}$ is a necessary but not sufficient condition for radiative $\mathrm{CP}$ violation. This is clearly illustrated in our discussion of the scalar potential above. For instance, in the MSSM case, if a $\Delta \lambda_{5}$ is taken as the only modification to the tree level parameters in Eq.(2), the constraint given by Eq.(15) is upset and the potential has no lower bound along $\cos \delta=\Omega$. An explicit calculation of the $3 \times 3$ physical Higgs mass squared matrix $m_{H i j}^{2}$ actually gives

$$
\operatorname{det}\left(m_{H}^{2}\right)=\lambda_{5}\left(p q-r^{2}\right) \sin ^{2} 2 \beta \sin ^{2} \delta
$$


which is negative. This inconsistency is also pointed out by Haba [11], who then suggested that it would be fixed when top/stop loop contributions to the other parameters, mainly $\lambda_{2}$, in the potential are included. In our opinion, it is at least of theoretical interest to see what the 1-loop gaugino/higgsino effect alone could do to the vacuum solution of a supersymmetric 2HDM. A consistent treatment of the 1-loop effect should of course take into consideration contributions to all the 10 parameters in the potential $V$. In Fig.1, we illustrate all the corresponding 1-loop diagrams involved. Obviously, $\lambda_{5}, \lambda_{6}, \lambda_{7}$ as well as (the sum of) $\lambda_{3}$ and $\lambda_{4}$ get finite contributions while the other five parameters receive divergent contributions. Note that in the diagrams, we do not distinguish $\Delta \lambda_{3}$ and $\Delta \lambda_{4}$; only their sum is involved in $\langle V\rangle$. Here we are not going to list all the tedious analytical expressions of these results, we plot the numerical results of major interest in Fig.2 instead. The plots are for the chargino contributions only, as functions of $m=M_{\tilde{g}} / \mu$, the gauginohiggsino mass ratio. Our results here presented give the 1-loop effect before EW symmetry breaking, i.e. mass mixing between the gaugino and higgsino were not considered. Further modifications due to the symmetry breaking are not expected to change the general features. Here, the neutralino part can simply be inferred from symmetry. For each charged fermion loop diagram, there is one from the neutral $S U(2)_{L}$ partner of identical amplitude. Then there is another one with the $S U(2)_{L}$ gaugino and the gauge coupling replaced by the $U(1)_{Y}$ ones.

Now we are in position to discuss the feasibility of radiative CP violation within a consistent approximation. In the model, $\Delta \lambda_{6}$ and $\Delta \lambda_{7}$ are identical. For most region of plot, they are of magnitude substantially larger than $\Delta \lambda_{5}$. A essential condition for SCPV is that $\Omega$, as given by Eq.(3), has magnitude less than one. Here the value of a divergent $\Delta m_{3}^{2}$ is also involved. If we take the finite result from $\overline{M S}$ scheme, for example, $\Delta m_{3}^{2}$ has a magnitude that increases fast with that of $m$ for $|m|>1$. Obviously, some fine tuning is needed to get $|\Omega|<1$, though a small window on $m$ always exists not too far from $|m|=1$, for each sign of $\mu$, for a not too small $\mu$. With EW-scale $\mu, \Delta m / m$ of the admissible regions are of order $10^{-2}$, though the severe fine tuning can be tamed by having small $\mu$. 
Apart from the small "pseudoscalar" mass objection, the admissible $\Omega$ region obviously gives phenomenologically unattractive $\mu$ and $M_{\tilde{g}}$ values. Renormalization for $m_{3}^{2}$ here could be tricky, as there is no definite guideline on what its renormalized value should be, especially in the CP violating case. In a softly broken SUSY model, such as MSSM, tree level value for $m_{3}^{2}$ is actually allowed, but then the $B$-term involved gives a negative contribution to $\Delta \lambda_{5}$. Apparently, one can take the renormalized value of $m_{3}^{2}$ as a free parameter and adjust it to give $|\Omega|<1$ for a fix $m$, at least when the $B$-term 1-loop effects are neglected. In any case, however, the fine tuning conclusion stands, as suggested by the Georgi-Pais theorem.

The case of our new model is relatively complicated. First of all, the gaugino/higgsino contributions are there before EW symmetry breaking, as presented above. In our new model, though the new quark doublet $Q$ has mass before EW symmetry breaking, a similar set of 1-loop diagrams, as those given in Fig.1, can only be completed when the EW breaking mass of the top and its mixing with $T$ are taken into consideration. But then the plausibly large Yukawa couplings give rise to substantial results. Otherwise, the qualitative features are similar to the gaugino/higgsino case, with the exception that here $\lambda_{3}+\lambda_{4}$ also receives logarithmic divergent contributions. In Fig.3, we presented some numerical results. The plots use again $m$ as parameter which in this case denotes $M_{Q} / v_{2}$. For simplicity, we assume $\lambda_{Q}=\lambda_{t}$. Note that around the $\mathrm{CP}$ violating vacuum of concern here, $\tan \beta \sim 1$ is to be expected in the SUSY case, as one can easily see by using Eqs.(11) and (12), with tree-level values of the various parameters assumed.

Here all the results are independent of the sign of $m . \Delta \lambda_{6}$ and $\Delta \lambda_{7}$ are different but always of the same signs. They are still of substantially larger magnitude when compared with $\Delta \lambda_{5}$, while the latter is of interesting value for small value of $m$, say $<1$. However, $\Delta m_{3}^{2}(\overline{M S})$ always has the opposite sign to that of $\Delta \lambda_{6}$ or $\Delta \lambda_{7}$, making a naive use of the value to fit in the $|\Omega|<1$ condition impossible. Hence, for SCPV to occur, a tree level $m_{3}^{2}$ value is needed. The general feature is independent of $\tan \beta$; but making its value really large suppresses $\Delta \lambda_{5}$ and is hence undesirable. Soft SUSY breaking also provides the natural source of a tree level $m_{3}^{2}$, while the $B$-term loop effects would be relatively 
negligible as they are suppressed by the small gauge couplings. While the $m_{3(\text { tree })}^{2}$ then has to be chosen to roughly match the $t$ - $T$ 1-loop effect, the large Yukawa couplings make this relatively natural, as a value of the order $v_{2}$ is all required. Accepting that, $\Delta m_{3(\text { tree })}^{2} / m_{3(\text { tree })}^{2}$ of the solution region is not quite small, $\gtrsim .35$ for $m \leq 1$, representing only a moderate fine tuning. The small $m_{3}^{2}$ required is natural, as its zero limit provides the scalar potantial with an extra Peccei-Quinn type symmetry. We expect a SUSY version of our model to be phenomenologically interesting. It has extra quarks close to the top mass scale and can give a feasible radiative $\mathrm{CP}$ violation scenario, though compatibility with top-phenomenology awaits detailed investigation.

Further discussions. An alternative, and may be more powerful, approach to the problem is to analyse the full 1-loop effective potential. Actually, we did check explicitly that we get the same numerical results for the modifications in all the potential parameters, from differentiating the standard Coleman-Weinberg expression of the 1-loop potentials for both cases above. With proper treatment of renormalizations for all the divergent quantities taken, the approach is expected to give a much better approximation to the quantum behavior of the potential and the physical Higgs masses. However, a detailed Coleman-Weinberg analysis of a 2HDM after symmetry breaking needed to be done more carefully, for instance, along the line suggested in Ref. [12].

Renormalization group runnings and contributions from other ingredients such as scalars should also be taken into consideration in the analysis of the detailed phenomenological implications and feasibility of a full model. The latter is especially important in the SUSY case. Recall that cancellations between superpartners is the natural mechanism that eliminates the quadratic divergence, as those arise from Fig.1(g) and (h), in $\Delta m_{1}^{2}$ and $\Delta m_{2}^{2}$. For $\Delta \lambda_{5}$ specifically, the squarks loop contributions are suppressed by the naturally heavier soft mass scale. Supersymmetric models with heavy fermion loop giving the necessary $\Delta \lambda_{5}$, however, would have problem suppressing the corresponding superparticle contributions. While the kind of detailed analysis for the MSSM is abundant [13], not much attention has been put into a $\mathrm{CP}$ violating scenario. The latter situation deserves more efforts. 
We have presented a consistent 1-loop analysis of the feasibility of radiatively induced SCPV, for both MSSM and our proposed new model. The latter is illustrated to have a good chance to be phenomenologically viable. We will report more on the topic in a forthcoming publication.

Acknowledgements. O.K. is indebted to P.H. Frampton for discussions and encouragement, and for suggestions on improving the manuscript. F.L. wants to thank L.N. Chang and T. Takeuchi for discussions in the early phase of this work. O.K. was supported by the U.S. Department of Energy under Grant DE-FG05-85ER-40219, Task B, and a UNC dissertation fellowship. F.L. was partly supported by the U.S. Department of Energy under Grant DE-FG05-92ER-40709, Task A. 


\section{REFERENCES}

[1] T.D. Lee, Phys. Rev. D8 (1973) 1226.

[2] S.L. Glashow and S. Weinberg, Phys. Rev. D15 (1977) 1958.

[3] G.C. Branco, Phys. Rev. D22 (1980) 2901.

[4] N. Maekawa, Phys. Lett. B282 (1992) 387.

[5] A. Pomarol, Phys. Lett. B287 (1992) 331.

[6] Georgi and Pais, Phys. Rev. D10 (1974) 1246.

[7] D. Chang and W.-Y. Keung, Phys. Rev. Lett. 74 (1995) 1928.

[8] J. Oliensis, Phys. Lett. 165B (1985) 99.

[9] See, for example, J.L. Hewett and T.G. Rizzo, SLAC-PUB-7430, hep-ph/9703337, and references therein.

[10] O.C.W. Kong, Mod. Phys. Lett. A11 (1996) 2547; Phys. Rev. D55 (1997) 383.

[11] N. Haba, Phys. Lett. B398 (1997) 305.

[12] E. Gildener and S. Weinberg, Phys. Rev. D13 (1976) 3333; K. Inoue, A. Kakuto, and Y. Nakano, Prog. Theor. Phys. 63 (1980) 234.

[13] See, for example, H.E. Haber and R. Hempfling, D48 (1993) 4280; H.E. Haber, R. Hempfling, and A.H. Hoang, CERN-TH-95-216, hep-ph/9609331; and references therein. 


\section{Figure Captions.}

Fig.1 : Gaugino/higgsino-loop diagrams giving rise to modifications to parameters in the potential. (Each dot indicates a helicity flip in the fermion propagator.)

Fig.2 : Plots of radiative correction from chargino loop verses $m\left(=M_{\tilde{g}} / \mu\right)$, with mass mixing from EW symmetry neglected. (a) $\Delta m_{3}^{2}(\overline{M S})$ in $25 \times g^{2} \mu^{2} / 16 \pi^{2}$; (b) $\Delta \lambda_{5}$; (c) $\Delta \lambda_{6}$ $\left(=\Delta \lambda_{7}\right) ;$ both of the latter curves with values in $g^{4} / 16 \pi^{2}$.

Fig.3 : Plots of radiative correction from $t$ - $T$ loop verses $m\left(=M_{Q} / v_{2}\right)$. (a) $\Delta m_{3}^{2}(\overline{M S})$ in $10 \times 3 \lambda_{t}^{2} v_{2}^{2} / 16 \pi^{2}$; (b) $\Delta \lambda_{5}$; (c) $\Delta \lambda_{6} ;$ (d) $\Delta \lambda_{7}$; all of the latter three curves with values in $3 \lambda_{t}^{4} / 16 \pi^{2} ;\left(\lambda_{t}=\lambda_{Q}, \tan \beta=1\right.$ assumed $)$. 


\section{FIGURES}

FIG. 1.

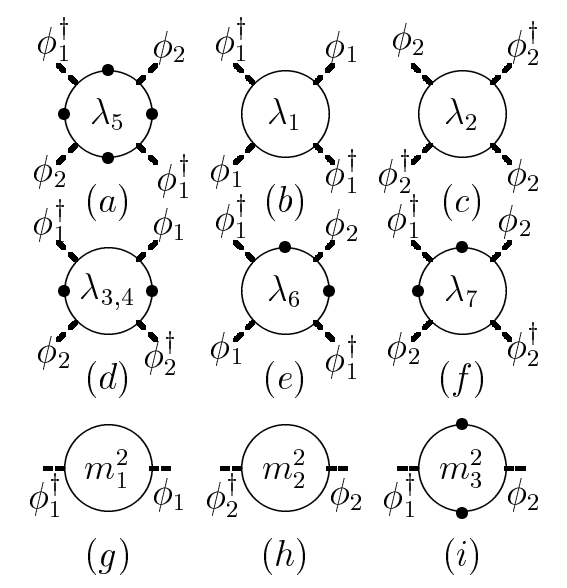


FIG. 2.

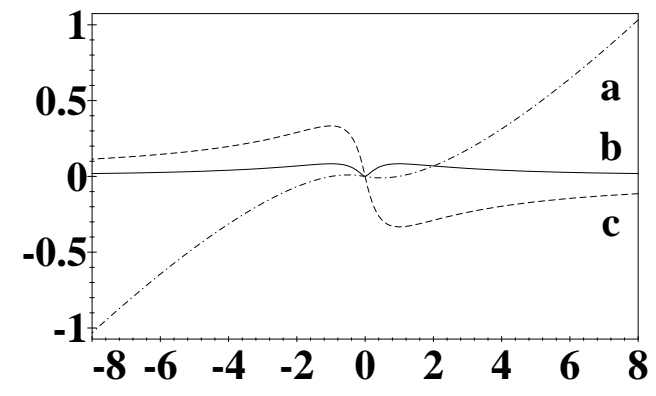


FIG. 3.

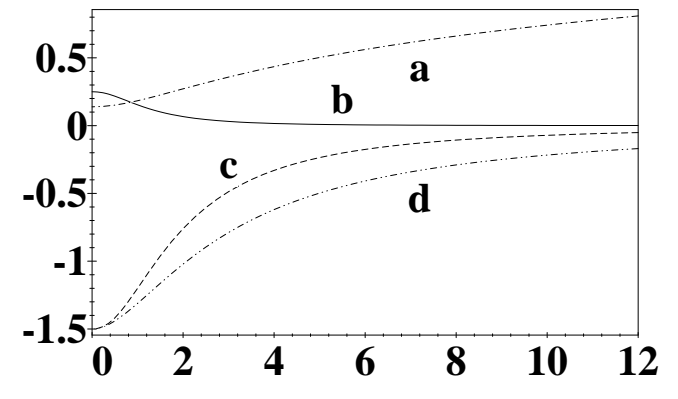

